OPEN ACCESS

Edited by:

Orhan Alimoglu,

Istanbul Medeniyet University Goztepe

Education and Research

Hospital, Turkey

Reviewed by:

Zahir Soonawalla,

Oxford University Hospitals NHS Trust,

United Kingdom

Kojiro Taura,

Kyoto University, Japan

Tomoaki Yoh,

Kyoto University, Japan

*Correspondence:

Hong Wu

wuhong7801@163.com

Genshu Wang

wgsh168@163.com

†These authors have contributed equally to this work

Specialty section

This article was submitted to

Surgical Oncology,

a section of the journal

Frontiers in Oncology

Received: 11 September 2019

Accepted: 24 March 2020

Published: 28 April 2020

Citation:

Li H, Li J, Wang J, Liu H, Cai B,

Wang $\mathrm{G}$ and Wu H (2020)

Assessment of Liver Function for

Evaluation of Long-Term Outcomes of

Intrahepatic Cholangiocarcinoma: A

Multi-Institutional Analysis of 620

Patients. Front. Oncol. 10:525.

doi: 10.3389/fonc.2020.00525

\section{Assessment of Liver Function for Evaluation of Long-Term Outcomes of Intrahepatic Cholangiocarcinoma: A Multi-Institutional Analysis of $\mathbf{6 2 0}$ Patients}

\author{
Hui $\mathrm{Li}^{1,2+}$, Jiaxin $\mathrm{Li}^{1,2 t}$, Jinju Wang ${ }^{1,2 \dagger}$, Hailing Liu ${ }^{1,2}$, Bole Cai ${ }^{1,2}$, Genshu Wang ${ }^{3 *}$ and \\ Hong $W u^{1,2 *}$
}

\begin{abstract}
'Department of Liver Surgery, Liver Transplantation Division, West China Hospital, Sichuan University, Chengdu, China, ${ }^{2}$ Laboratory of Liver Surgery, West China Hospital, Sichuan University, Chengdu, China, ${ }^{3}$ Department of Hepatic Surgery and Liver Transplantation Center, The Third Affiliated Hospital of Sun Yat-sen University, Guangzhou, China
\end{abstract}

Background: Liver function is a routine laboratory test prior to curative liver resection. It remains unclear whether the albumin-bilirubin (ALBI) grade and albumin-to-alkaline phosphatase ratio (AAPR) can predict long-term outcomes of surgically treated patients with intrahepatic cholangiocarcinoma (ICC).

Methods: This study investigated the correlation between ALBI grade and AAPR with overall survival (OS) after liver resection and then compared their accuracy to the Child-Pugh score. Harrell's concordance index (C-index) and Akaike information criterion (AIC) were used to compare accuracy of models.

Results: A total of 620 ICC patients were included, 477 in derivation cohort and 143 for validation. 0.348 was identified as the cutoff value for AAPR after calculating the Youden index. In the derivation cohort, elevated ALBI grade was associated with worse prognosis [hazard ratio (HR): 1.751, 95\% confidence interval (Cl): 1.329 to 2.306], and a decreased AAPR value was correlated with shorter OS (HR: 1.969, 95\% Cl: 1.552 to 2.497). Multivariate analysis suggested that the ALBI grade, AAPR, CA19-9, tumor number, and microvascular invasion were independent prognostic predictors for OS. ALBI grade and AAPR showed more accuracy in evaluating OS for surgically treated ICC patients than the Child-Pugh score (C-index: 0.559, 0.600 vs. 0.528; AIC: 3023.84, 3007.73 vs. 3034.66). Our findings were validated in an independent cohort from another clinical center.

Conclusions: Importantly, the ALBI grade and AAPR showed greater discriminatory power than the Child-Pugh score in assessing long-term outcomes following hepatectomy for ICC. The AAPR was more accurate than the ALBI grade. It was beneficial to consider the ALBI grade and AAPR as useful surrogate markers to identify patients at risk of poor postoperative outcomes.

Keywords: intrahepatic cholangiocarcinoma, albumin-bilirubin grade, albumin-to-alkaline phosphatase ratio, surgery, prognostic utility 


\section{INTRODUCTION}

Liver cancer remains the sixth most commonly diagnosed cancer as well as the fourth leading cause of malignancyrelated mortality worldwide, which gives rise to nearly 841,000 new cases and 782,000 deaths every year (1). Intrahepatic cholangiocarcinoma (ICC) is one of the subtypes of cholangiocarcinoma, which accounts for about $10-15 \%$ of all primary hepatic malignancies1 $(2,3)$. The incidence and mortality of ICC rank only behind hepatocellular carcinoma (HCC) and increase aggressively in recent years (4). In contrast to HCC, ICC is a cancer associated with rapid progression and a dismal 5 -year survival rate $<5-10 \%(5)$. Surgery remains the most efficient treatment for patients with early-stage tumor (6). However, prognosis after hepatic resection is still unsatisfactory with a 5 -year survival rate $<40 \%$ for ICC patients with R0 resection $(6,7)$. Systematic chemotherapy based on gemcitabine and cisplatin has become standard strategy for advanced or metastasis ICC, but the median survival time is $<1$ year (6). Therefore, it is necessary to explore useful prognostic factors that facilitate the selection of appropriate surgical candidates and therapeutic strategies.

The 7th edition of the American Joint Committee on Cancer (AJCC) Staging Manual introduced a new staging system, which has become the most commonly used for ICC $(8,9)$. In the current 8 th edition, the $\mathrm{T} 1$ category has been subdivided to $\mathrm{T} 1 \mathrm{a}$ and T1b according to a maximum tumor diameter of $5 \mathrm{~cm}$ (10). Wang et al. proposed a prognostic nomogram, comprising serum carcinoembryonic antigen (CEA), CA 19-9, tumor number and diameter, vascular invasion, lymph node, and local extrahepatic metastasis, for ICC that underwent partial hepatectomy (3). Apart from tumor staging systems, serum parameters and prognostic models are explored in the assessment of prognosis of ICC. Tumor markers, such as CEA and CA19-9, have been confirmed for their value in predicting prognosis of ICC patients $(11,12)$. Inflammationbased prognostic models such as neutrophil-to-lymphocyte ratio (NLR) (13), platelet-to-lymphocyte ratio (PLR) (14), and lymphocyte-to-monocyte ratio (LMR) (13) have been introduced to evaluate prognosis. Moreover, the underlying liver function also plays an important role in prognostic outcomes of ICC patients. Generally, the Child-Pugh grade is used to evaluate hepatic function reserve prior to hepatectomy. It has been reported to be an independent prognostic factor for overall survival (OS) for ICC (15). Recently, the albumin-bilirubin (ALBI) grade and albumin-to-alkaline phosphatase ratio (AAPR) were introduced to assess liver function. Several studies have investigated their prognostic value in HCC, demonstrating that decreased AAPR and elevated ALBI grade were associated with worse prognosis after hepatic resection (16-18). However, their application value in predicting prognosis of ICC patients remains unclear.

In the present study, we aimed to assess the prognostic significance of scoring systems based on preoperative liver function for surgically treated ICC and then propose simple and feasible models to stratify patients at different risk of postoperative outcomes.

\section{METHODS}

\section{Study Population}

In this multicenter retrospective study, a total of 620 surgically treated ICC patients at West China Hospital and the Third Affiliated Hospital of Sun Yat-sen University were consecutively included. Patient set from the West China Hospital between January 2009 and December 2017 were used to explore our initial practice (derivation cohort) and then the findings were verified in an independent cohort from the Third Affiliated Hospital of Sun Yat-sen University (located in southern China, validation cohort). All the enrolled patients were newly pathologically diagnosed ICC and underwent surgical resection with curative intent for first time. The exclusion criteria were as follows: patients received radiofrequency ablation, transarterial chemoembolization, chemotherapy, or other anticancer therapies before hepatectomy; patients with extrahepatic metastasis; patients who underwent surgical resection for tumor rupture; and those without complete medical records. All eligible patients or their relative provided written informed consent. This study was approved by the Ethics Committee of the West China Hospital and the Third Affiliated Hospital of Sun Yatsen University, in accordance with the guidelines of the 1975 Declaration of Helsinki (19).

\section{Clinical Data Collection and Follow-Up}

All the patient information and preoperative laboratory parameters were reviewed and retrieved from the hospital electronic or handwritten medical records. The ALBI was calculated by the formula ( $\log _{10}$ bilirubin $\left.\times 0.66\right)-($ albumin $\times$ 0.085 ), where bilirubin was in $\mathrm{mol} / \mathrm{L}$ and albumin was in $\mathrm{g} / \mathrm{L}$ (20). Patients were classified according to the previously reported cutoffs $\leq-2.60$ (ALBI grade 1$),>-2.60$ to $\leq-1.39$ (ALBI grade 2 ), and $\geq-1.39$ (ALBI grade 3 ). AAPR was calculated from dividing the ALB level by serum ALP level, where ALB was in $\mathrm{g} / \mathrm{L}$ and ALP was in U/L (16). Patients were also stratified according to the cutoffs. Moreover, tumor-related clinicopathological characteristics including differentiation, number of tumor nodules, maximum tumor diameter, macrovascular invasion, microvascular invasion (MVI), and extrahepatic metastasis were also acquired. MVI was defined as histologically identified vascular invasion of small vessels, while macrovascular invasion was defined as radiologically diagnosed vascular invasion of large vessels or macroscopic thrombosis. The TNM stages were stratified according to the 8th edition of the AJCC Staging Manual. Patients were followed up according to National Comprehensive Cancer Network (NCCN), regularly contrastenhanced ultrasonography per month at first year, and then every 3 months for 2 years, and then every 6 months thereafter. Besides, we contact those who determined not to go back to the hospital to reexamination through telephone follow-up survey. Few patients (seven in West China Hospital and four in the Third Affiliated Hospital of Sun Yat-sen University) lost to follow-up at the end of follow-up in December 2018. Additionally, five patients (four in West China Hospital and one in the Third Affiliated Hospital of Sun Yat-sen University) were excluded due to incomplete medical records (three without 
TABLE 1 | Baseline characteristics of patients.

\begin{tabular}{|c|c|c|}
\hline Variables & $\begin{array}{l}\text { Derivation cohort } \\
\quad(n=477)\end{array}$ & $\begin{array}{l}\text { Validation cohor } \\
\qquad(n=143)\end{array}$ \\
\hline \multicolumn{3}{|c|}{ Patient factors/Laboratory parameters } \\
\hline Age [year, median (IQR)] & $58(49.5-64)$ & $59(51-67)$ \\
\hline Male gender, $n(\%)$ & $227(47.6)$ & $83(58)$ \\
\hline HBsAg [positive, $n(\%)]$ & $139(29.1)$ & $35(24.6)$ \\
\hline $\mathrm{HCV}, n(\%)$ & $2(0.4)$ & $2(1.4)$ \\
\hline Hepatolithiasis, $n$ (\%) & $83(17.4)$ & $12(8.4)$ \\
\hline \multicolumn{3}{|l|}{ Child-Pugh score, $n(\%)$} \\
\hline 5 & 407 (85.3) & $125(87.4)$ \\
\hline 6 & $66(13.8)$ & 15 (10.5) \\
\hline 7 & $7(0.4)$ & $3(2.1)$ \\
\hline Ascites, $n(\%)$ & $47(9.9)$ & $5(3.5)$ \\
\hline ALB [g/L, mean (SD)] & $42.7(4.6)$ & $41.7(4.1)$ \\
\hline TBIL [ $\mu \mathrm{mol} / \mathrm{L}$, mean (SD)] & $14.3(8.5)$ & $14.2(7.7)$ \\
\hline ALP [U/L, mean (SD)] & $136.6(95.1)$ & $135.3(76.1)$ \\
\hline \multicolumn{3}{|l|}{ ALBI grade, $n(\%)$} \\
\hline 1 & $387(81.1)$ & $103(72)$ \\
\hline 2 & 90 (18.9) & $40(28)$ \\
\hline \multicolumn{3}{|l|}{ AAPR grade, $n(\%)$} \\
\hline $1(>0.348)$ & $293(61.4)$ & $76(53.1)$ \\
\hline $2(\leq 0.348)$ & $184(38.6)$ & 67 (46.9) \\
\hline \multicolumn{3}{|l|}{ CA19-9 } \\
\hline$<22, n(\%)$ & $137(28.7)$ & $37(25.9)$ \\
\hline$\geq 22, n(\%)$ & $330(69.2)$ & $105(73.4)$ \\
\hline Not available & $10(2.1)$ & $1(0.7)$ \\
\hline \multicolumn{3}{|c|}{ Histological and gross features of tumors } \\
\hline Tumor size [cm, mean (SD)] & $6(2.7)$ & $6.1(2.4)$ \\
\hline Solitary tumor, $n$ (\%) & 337 (70.6) & $88(61.5)$ \\
\hline Tumor differentiation & $17 / 55$ & $10 / 67$ \\
\hline Well & $12(2.5)$ & $5(3.5)$ \\
\hline Moderate & $362(75.9)$ & $113(79)$ \\
\hline Poor & $78(16.4)$ & $22(15.4)$ \\
\hline Not available & $25(5.2)$ & $3(2.1)$ \\
\hline Macrovascular invasion, $n(\%)$ & $106(22.2)$ & $52(36.4)$ \\
\hline Microvascular invasion, $n(\%)$ & $51(10.7)$ & $14(9.8)$ \\
\hline Cirrhosis, $n$ (\%) & $136(28.5)$ & $20(14)$ \\
\hline
\end{tabular}

Overall survival, months, mean $\quad 24.9(23.0,26.8) \quad 30.5(26.7,34.2)$ (95\% Cl)

$A L B I$, albumin-bilirubin; $A A P R$, albumin-to-alkaline phosphatase ratio; $A L B$, albumin; TBIL, total bilirubin; ALP, alkaline phosphatase; CA19-9, carbohydrate antigen 19-9; IQR, interquartile range; $S D$, standard deviation; $\mathrm{Cl}$, confidence interval.

intraoperative outcomes and two without preoperative liver function test).

\section{Statistical Analysis}

The statistical analyses were performed by using the software of SPSS (version 22.0, Chicago, IL, USA), MedCalc (version 15.2.2.0, Ostend, Belgien), and GraphPad Prism (version 8.0, San Diego, California, USA). Harrell's concordance index (C-index) and Akaike information criterion (AIC) were calculated by using R (https://www.r-project.org/, v3.3.4). Receiver-operating characteristic (ROC) curves were applied to determine the optimal cutoff value as the Youden index attained maximum value. Pearson's chi square test and Student's $t$-test were used to investigate the correlation of categorical and continuous variables to AAPR level, respectively. Kaplan-Meier curves were plotted for derivation and validation cohort according to each cutoff value, and their differences were tested using log-rank test. Those clinicopathological parameters with $P<$ 0.2 in the univariable Cox proportional hazards regression were considered for generating multivariable Cox regression (enter method) to identify potential independent prognostic factors for OS (21). A two-tailed $P<0.05$ was considered statistically significant.

\section{RESULTS \\ Derivation Cohort}

In the derivation cohort, 477 patients [227 (47.6\%) male; median (IQR) age, $58(49.5,64)$ years] were finally recruited after excluding the only one with preoperative liver function of ALBI grade 3 . Table 1 summarized baseline characteristics of the patient cohorts. No patient was stratified into Child-Pugh grade C; 7 (0.8\%) were Child-Pugh B. Among patients with ChildPugh A, 407 (85.3\%) were 5 points, whereas 66 (13.8\%) were 6 points. A cutoff value of 0.348 for AAPR was identified by using ROC. A total of $292(61.2 \%)$ patients with AAPR value more than 0.348 were classified into elevated AAPR group (AAPR grade $1)$, whereas $185(38.8 \%)$ were stratified into decreased group (AAPR grade 2). By using ALBI grade, 387 (81.1\%) patients were stratified into ALBI grade 1 and 90 (18.9\%) were stratified in ALBI grade 2. Eighty-three (17.4\%) patients also suffered from hepatolithiasis. The carbohydrate antigen 19-9 (CA19-9) was available for 467 patients. Since the reference range was $22 \mathrm{U} / \mathrm{ml}$, elevated serum CA19-9 was detected in $69.2 \%$ of the patients.

The correlation between patient characteristics with ALBI and AAPR were summarized in Table 2. A higher ratio of Child-Pugh score 5 and larger tumor size were observed in ALBI grade 1 and AAPR grade 1. The mean OS was 24.9 months, $203(42.6 \%)$ survived at the end of followup, whereas $274(57.4 \%)$ died. A comparison of ALBI and AAPR values between survival and death demonstrated a decreased AAPR value and increased ALBI value in the dead group (Figures 1A,B). In patients with Child-Pugh $A$, an elevated AAPR value and decreased ALBI were observed in patients with Child-Pugh score 5 (Figures 1C,D). KaplanMeier analyses with log-rank test suggested that ALBI grade 1, elevated AAPR value, normal serum CA19-9 level, and solitary tumor correlated with better prognosis, whereas patients with microvascular invasion, cirrhosis, poor tumor differentiation, and Child-Pugh score 6 were related to poor survival outcomes (Figures 2A,B and Supplementary Figure 1). Among 19 clinicopathological characteristics, 14 were identified as potentially relevant with $P<0.2$ in the univariate Cox regression analyses. Twelve of them were introduced in consequent 
TABLE 2 | Correlation of characteristics with the ALBI and AAPR of 477 ICC patients treated with surgical resection in the derivation cohort.

\begin{tabular}{|c|c|c|c|c|c|c|}
\hline \multirow[t]{2}{*}{ Characteristics } & \multicolumn{3}{|c|}{ ALBI } & \multicolumn{3}{|c|}{ AAPR } \\
\hline & Grade $1(n=387)$ & Grade $2(n=90)$ & $\boldsymbol{P}$ value & $>0.348(n=293)$ & $\leq 0.348(n=184)$ & $P$-value \\
\hline Age [year, mean (SD)] & $57.1(10.7)$ & $56.2(10.9)$ & 0.958 & $56.9(10.9)$ & $56.9(10.4)$ & 0.998 \\
\hline Male gender, $n(\%)$ & $175(45.2)$ & $52(57.8)$ & 0.032 & $143(48.8)$ & $84(45.7)$ & 0.511 \\
\hline HBsAg [positive, $n(\%)]$ & $106(27.5)$ & $33(37.1)$ & 0.091 & $106(36.6)$ & $33(18.0)$ & $<0.001$ \\
\hline Hepatolithiasis, $n$ (\%) & $61(15.8)$ & $22(24.4)$ & 0.061 & $40(13.7)$ & $43(23.4)$ & 0.006 \\
\hline \multicolumn{7}{|l|}{ Child-Pugh score, $n$ (\%) } \\
\hline 5 & $348(89.9)$ & $59(65.5)$ & $<0.001$ & $265(90.4)$ & $142(77.2)$ & $<0.001$ \\
\hline 6 & $39(10.1)$ & $27(30.0)$ & & $27(9.2)$ & $39(21.2)$ & \\
\hline Ascites, $n(\%)$ & $36(9.3 \%)$ & $11(12.2)$ & 0.432 & $24(8.2)$ & $23(12.5)$ & 0.152 \\
\hline ALB [g/L, mean (SD)] & $43.9(2.9)$ & $36.6(3.1)$ & $<0.001$ & $43.5(3.5)$ & $41.2(4.6)$ & $<0.001$ \\
\hline TBIL [ $\mu \mathrm{mol} / \mathrm{L}$, mean (SD)] & $13.6(5.7)$ & $17.1(15.4)$ & 0.001 & $13.8(5.8)$ & $15.1(11.6)$ & 0.102 \\
\hline ALP [U/L, mean (SD)] & $128.1(85.1)$ & $172.7(123.8)$ & $<0.001$ & $88.7(23.1)$ & $212.6(114.8)$ & $<0.001$ \\
\hline \multicolumn{7}{|l|}{ CA19-9 } \\
\hline$<22, n(\%)$ & $114(30.2)$ & $23(25.8)$ & 0.439 & $93(32.4)$ & $44(24.4)$ & 0.075 \\
\hline$\geq 22, n(\%)$ & $264(69.8)$ & $66(74.2)$ & & $194(67.6)$ & $136(75.6)$ & \\
\hline Tumor size [cm, mean (SD)] & $5.8(2.5)$ & $6.6(3.3)$ & 0.014 & $5.5(2.3)$ & $6.8(3.2)$ & $<0.001$ \\
\hline Solitary tumor, $n$ (\%) & $276(71.3)$ & $61(67.8)$ & 0.536 & $217(74.1)$ & $120(65.2)$ & 0.113 \\
\hline \multicolumn{7}{|l|}{ Tumor differentiation } \\
\hline Well & $10(2.6)$ & $2(2.2)$ & 0.068 & $10(3.4)$ & $2(1.1)$ & 0.022 \\
\hline Moderate & $302(78.0)$ & $60(66.7)$ & & $219(74.7)$ & $143(77.7)$ & \\
\hline Poor & $55(14.2)$ & $23(25.6)$ & & $54(18.4)$ & $24(13)$ & \\
\hline Macrovascular invasion, $n$ (\%) & $82(21.2)$ & $24(26.7)$ & 0.324 & $50(17.1)$ & $56(30.4)$ & 0.001 \\
\hline Microvascular invasion, $n(\%)$ & $43(11.1)$ & $8(8.9)$ & 0.579 & $29(9.9)$ & $22(12.0)$ & 0.550 \\
\hline Cirrhosis, $n(\%)$ & $102(26.4)$ & $34(37.8)$ & 0.038 & $90(30.7)$ & $46(25)$ & 0.211 \\
\hline Overall survival, months, mean $(95 \% \mathrm{Cl})$ & $25.6(23.5,27.6)$ & $21.9(16.9,26.9)$ & & $27.4(24.9,29.8)$ & $20.9(18.1,23.9)$ & \\
\hline
\end{tabular}

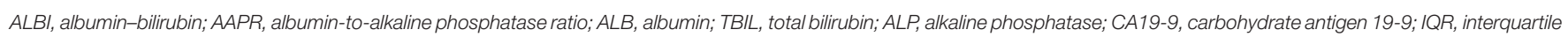
range; $S D$, standard deviation; $\mathrm{Cl}$, confidence interval.

multivariate Cox proportional hazards regression model, and 6 were identified as independent prognostic predictors (Figure 2E and Supplementary Table 2).

In the subgroup analysis of patients with Child-Pugh score 5 , the prognostic outcome was further analyzed according to ALBI and AAPR grades. Three hundred forty-nine (85.7\%) were stratified as ALBI grade 1 and 266 (65.4\%) were classified into AAPR grade 1. Consistently, better prognosis was also found in patients with ALBI grade 1 and AAPR value more than 0.348 (Figure 3).

C-index and AICs were also compared between ALBI grade and AAPR with Child-Pugh score in predicting survival outcomes. The results suggested that the ALBI grade and AAPR were more representative in evaluating OS for surgically treated ICC patients than Child-Pugh score (C-index: $0.559,0.600$ vs. 0.528; AIC: $3023.84,3007.73$ vs. 3034.66) (Table 3). Moreover, the AAPR showed more accurate predicting value than the ALBI grade.

In addition, we also compared perioperative outcomes between ICC patients in different ALBI and AAPR groups. The results showed similar operative time, estimated blood loss, blood transfusion, postoperative complications, and hospitalization between ICC patients with different preoperative ALBI score and
AAPR levels (Supplementary Table 4). There was a tendency that patients with elevated AAPR levels correlated with decreased frequency of postoperative complications with a $P<0.1$.

\section{Validation Cohort}

Consecutive ICC patients treated with surgical resection at the Third Affiliated Hospital of Sun Yat-sen University from January 2011 to December 2017 were recruited to validate our findings. After the exclusion of one patient with preoperative ALBI grade 3, 143 patients [83 (58\%) male, median (IQR) age, $59(51,67)$ years] were included. The correlation of tumor characteristics with ALBI grade and AAPR grade is shown in Supplementary Table 1. Consistent with derivation cohort, patients in ALBI grade 1 and AAPR grade 1 correlated with an increased ratio of Child-Pugh score 5 and mean OS.

A total of 125 patients $(87.4 \%)$ were associated with ChildPugh score 5. By calculating ALBI score and AAPR values, $103(72 \%)$ were stratified into ALBI grade 1 and 76 (53.1\%) patients have an AAPR value more than 0.348. The mean OS was 30.5 months. In the univariate analyses, Child-Pugh score, ALP, ALBI grade, AAPR, CA19-9, tumor number, and microvascular invasion were possible variables that correlated with patients' prognosis (Figures 2C,D). The ALBI grade, AAPR, 

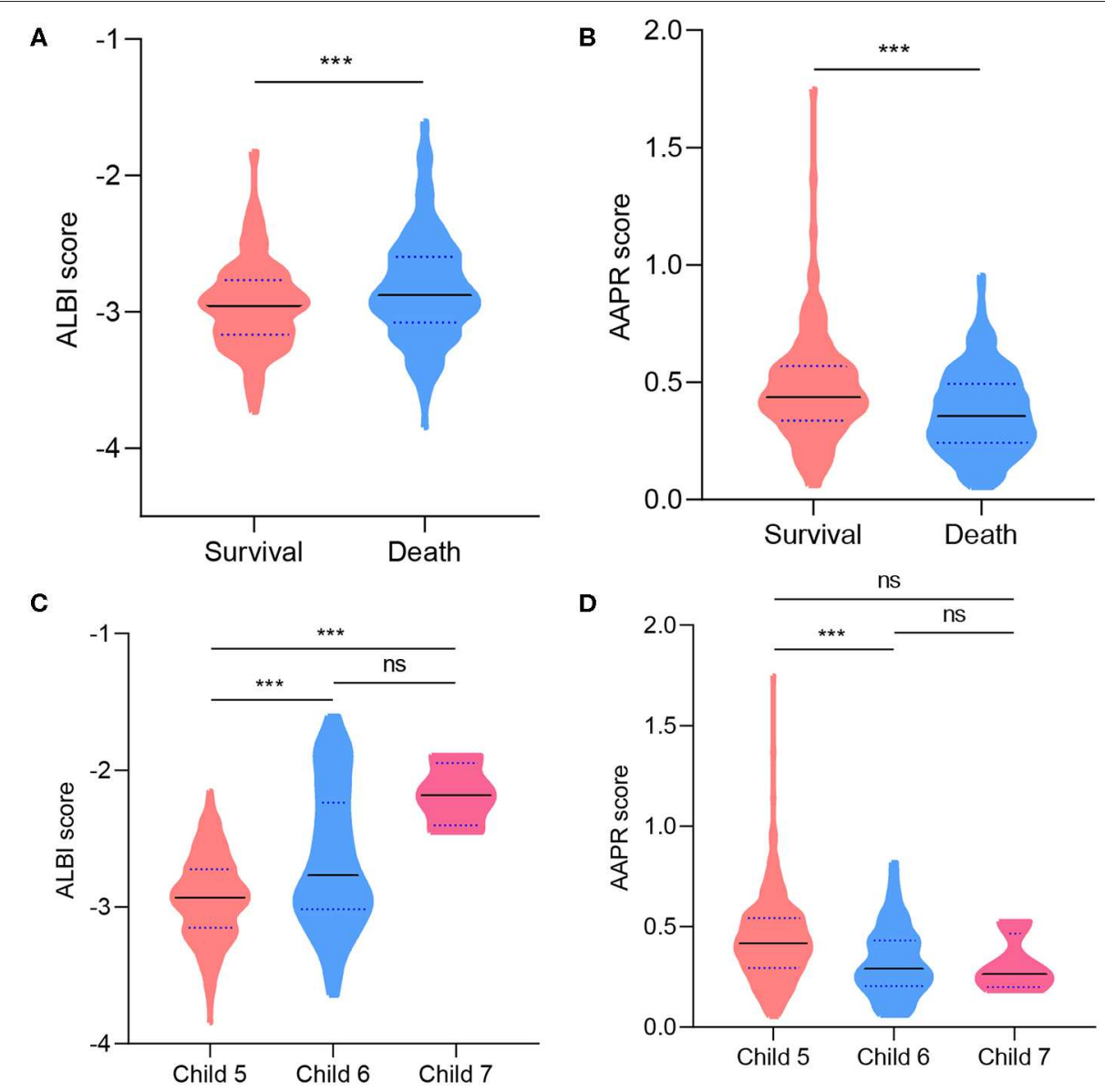

FIGURE 1 | Violin plots showing the distribution of ALBI and AAPR score: in survival and death group at the end of follow-up (A,B); for patients with different Child-Pugh scores (C,D). Solid lines represent median value; dotted lines represent quartiles. ${ }^{\star} P<0.05$; ${ }^{* \star} P<0.01$; ${ }^{\star \star \star} P<0.001$; ns, not significant.

CA19-9, tumor number, and microvascular invasion were identified as independent prognostic predictors for postoperative survival (Figure 2F and Supplementary Table 2). The subgroup analyses of ICC patients in the validation cohort are shown in Supplementary Figure 2.

The short-term outcomes were also comparable between 143 ICC patients in different ALBI and AAPR groups from the Third Affiliated Hospital of Sun Yat-sen University (Supplementary Table 4).

\section{Discussion}

In the present study, we evaluated the potential impact of scoring systems based on preoperative liver function on prognostic outcomes in surgically treated ICC. Child-Pugh score and ALBI and AAPR grade showed their prognostic value in predicting OS. The multivariate analysis identified key variables that influenced OS, including tumor features (CA19-9, tumor number, and microvascular invasion) and liver function (ALBI and AAPR grade). These results were in accordance to previous studies (22).

In contrast to tumor characteristics, the underlying liver function is routinely detected prior to operation. As is well-known, preoperative liver function has an impact on postoperative outcomes for patients undergoing curative resection. Child-Pugh score is one of the most widely used for assessing liver function. It is also an indication for selecting proper candidate for surgical treatments. However, there are several limitations, including arbitrary cutoff points for laboratory indices and subjective clinical assessment of ascites and hepatic encephalopathy $(23,24)$. The ALBI grade, proposed by Johnson and colleagues, calculated from albumin and bilirubin, has been demonstrated to be a useful prognostic predictor for patients undergoing liver resection $(17,25)$. Serum ALB, a kind of protein synthesized in liver, is an indicator that reflects the protein synthetic capability. Moreover, it remains a modulator for inflammatory response, which is essential in retardation of liver cancer (26). In the univariate analyses, ALB was a potential factor relating to OS. However, as a component of ALBI, it had to be excluded in multivariate analysis. Our results were consistent with previous studies, validated the accurate prognostic value of ALBI grade for surgically treated ICC patients. Recently, Tsilimigras et al. demonstrated that the ALBI score was associated with both short- and long-term outcomes following hepatectomy for ICC and could prove a useful surrogate marker to identify patients at risk for adverse 

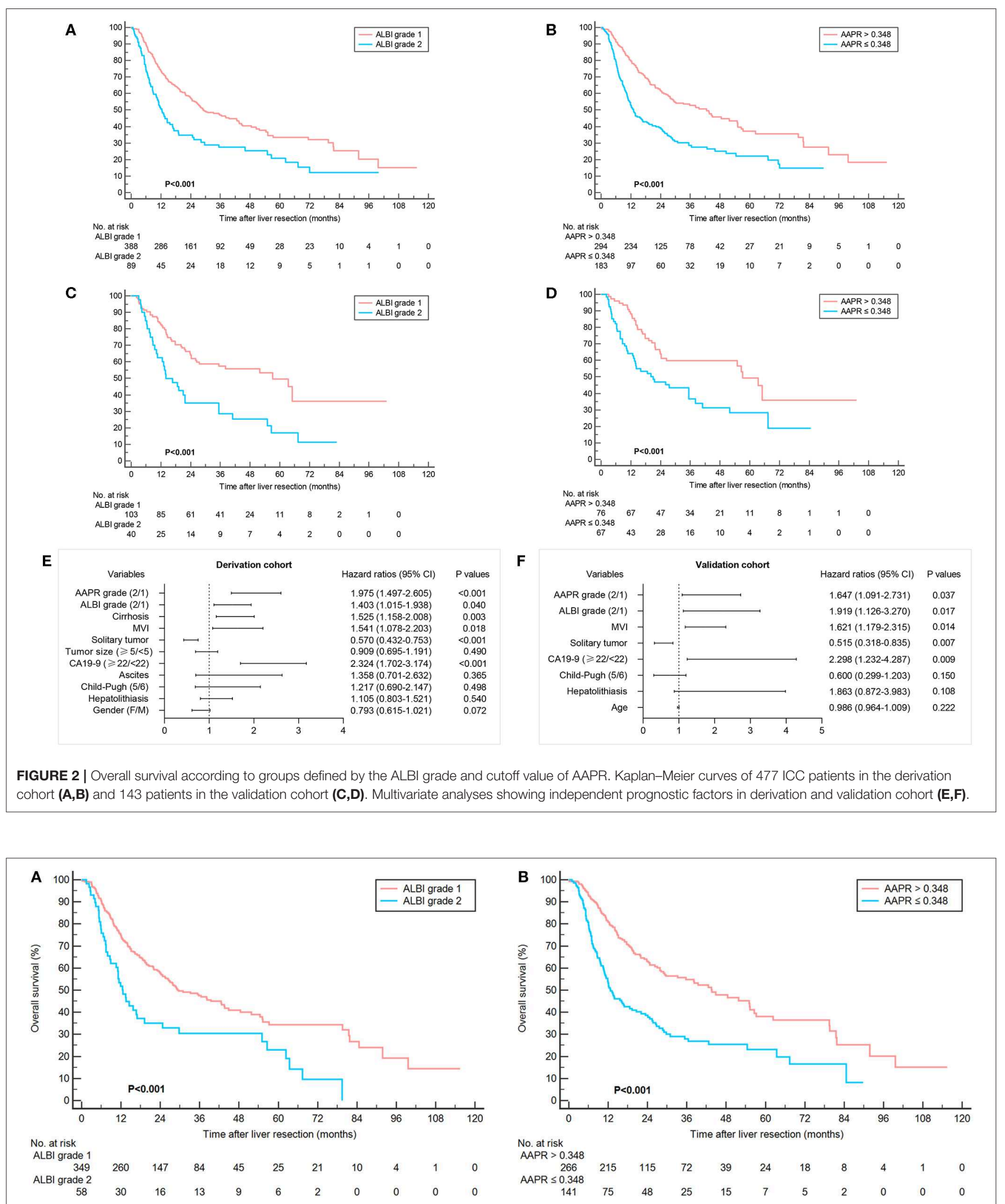

FIGURE 3 | Kaplan-Meier curves showing overall survival according to ALBI (A) and AAPR grade (B) in 407 patients with Child-Pugh score 5. 
TABLE 3 | Comparisons of the value of three models in predicting prognosis of overall survival among the patients in derivation and validation cohort.

\begin{tabular}{lcccc}
\hline Models & C-index & 95\% Cl & P-value & AIC \\
\hline $\begin{array}{l}\text { Derivation cohort } \\
\text { Child-Pugh score }\end{array}$ & 0.528 & $0.504-0.553$ & Ref & 3034.66 \\
ALBI grade & 0.559 & $0.532-0.586$ & 0.023 & 3023.84 \\
AAPR grade & 0.601 & $0.570-0.632$ & $<0.001$ & 3007.73 \\
Validation cohort & & & & \\
Child-Pugh score & 0.533 & $0.489-0.705$ & Ref & 710.09 \\
ALBI grade & 0.585 & $0.531-0.639$ & 0.039 & 702.20 \\
AAPR grade & 0.600 & $0.545-0.655$ & 0.012 & 700.61 \\
\hline
\end{tabular}

$A L B I$, albumin-bilirubin; AAPR, albumin-to-alkaline phosphatase ratio; Cl, confidence interval; Ref, reference; AIC, Akaike information criterion. A lower AIC, indicating lower loss of information, was more representative of the model.

outcomes (27). However, in our study, we found that the perioperative outcomes were comparable between different ALBI and AAPR grades in both derivation and validation cohort.

Initially, only ALB, ALP, and TBIL were put into multivariate analysis (Supplementary Table 3). The results showed that ALB and ALP were independent prognostic factors, whereas TBIL was not. Thus, we combined ALB and ALP to create a stronger prognostic factor-AAPR and compared its accuracy to ALBI and Child-Pugh classification. The AAPR grade based on a cutoff value of 0.348 was also an independent prognostic predictor for OS. Calculated from dividing the ALB by ALP, it was introduced by Chan et al. initially to predict prognosis of HCC patients who underwent surgical resection and palliative therapy (16). Cai et al. validated the prognostic value of AAPR in advanced HCC patients who did not receive any standard anti-cancer treatments (28). The serum ALP, another one of the most routinely detected parameters in laboratory test, is a hydrolase enzyme that contains a mixture of ALP isoenzymes from liver, bones, kidney, and placenta. Several studies have reported that the ALP level increases during childhood and other diseases such as hepatic diseases, osteomalacia, and bone tumor $(29,30)$. Patients with biliary diseases are always associated with elevated serum ALP level. Previous studies have reported that ALP was an independent prognostic factor for ICC patients $(31,32)$. Zhang et al. have evaluated the prognostic effect of serum liver enzymes in ICC patients and demonstrated that both ALB and ALP were predictive factors (31). However, the enrolled 173 patients were associated with locally advanced or metastatic tumors. In our study, 620 patients from two centers were associated with earlystage ICC and underwent curative resection, which remained the most efficient treatment for ICC patients. We also examined the prognostic significance of ALB and ALP either alone or in combination, and found that ALBI and AAPR showed a promising effect on stratifying ICC patients into a different risk of postoperative outcomes. They might show a stronger prognostic significance than ALB, ALP, and TBIL alone. Our results did not conflict with these studies, showed ALP as a potential relevant in the univariate analysis. It was also excluded in multivariable analysis. Our results also identified the decreased AAPR score as an independent risk factor of postoperative survival for surgically treated ICC patients.

Importantly, our present analysis suggested that the ALBI grade and AAPR score exhibited a greater discriminatory power than Child-Pugh score. Previous studies demonstrated at least equal discriminatory power, in prognostic terms, of ALBI score to Child-Pugh score (33). Apart from containing two subjective parameters (encephalopathy and ascites), Child-Pugh score is advocated for evaluating liver function with the precondition that patients suffered from cirrhosis. However, only a few ICC patients had cirrhosis, with a proportion of $25.2 \%$ in our current study. Therefore, it was not a surprise that the Child-Pugh score was not found to be an independent factor with respect to postoperative survival. To compare the accuracy of these three models, subgroup analysis was performed to investigate the performance of AAPR and ALBI grade within the ChildPugh score 5. The result showed distinct prognosis for patients with different AAPR and ALBI grade, indicating their potential advantages over Child-Pugh classification. Indeed, the degree of liver functional reserve varied greatly among patients with same Child-Pugh score. For patients with severe cirrhosis, their ChildPugh score might be the same as those with very early stage tumors. Consistent with previous studies, ALBI grade exhibited a greater discriminatory power than Child-Pugh score (33-35). Furthermore, consistent with ALBI grade, AAPR score showed a greater stratifying ability in representing liver function reserve for ICC patients treated with surgical resection.

ALBI and AAPR are easily accessible laboratory tests that are routinely detected for patients with liver diseases. Additionally, unlike other serodiagnosis or iconographical detections, they are low-cost tests that will not increase the total medical costs. However, it should be noted that the ALBI and AAPR scores are not tumor staging systems since they only measure preoperative liver function, taking no account of tumor-related factors. Further models combining ALBI, AAPR, and other tumor characteristics (tumor size, number, microvascular invasion status, and CA19-9) are in need to evaluate prognostic outcomes for surgically treated ICC patients.

There were several limitations that warrant consideration when interpreting our findings. Firstly, although our initial findings were validated by an independent cohort from different areas inside our country, it was limited by its retrospective design. Secondly, the etiologies for ICC differed from different geographic regions (especially Asia with Europe and America); further prospective international multicenter studies were required to verify our primary findings. In addition, in these two centers, to ensure patients' safety, part of patients received surgical resection after treating with hepatic protectants. Hence, few patients with Child-Pugh grade B were included. Additionally, the mechanisms underlying why liver function impact survival outcomes were not analyzed in the present study. In the future, we will perform a basic research to investigate the possible mechanisms using animal models and in vitro cell experiments. Finally, owing to insufficient ward beds, prompt surgical treatments were not available for a subset of ICC patients with operative indication; the referral bias could not be completely avoided. 


\section{CONCLUSIONS}

The present study provided compelling evidence that the AAPR score and ALBI grade were validated prognostic factors for surgically treated ICC patients. They enabled dividing these patients into independent groups before surgical treatment. Since three widely available laboratory parameters were involved, the AAPR score and ALBI grade were more objective, low-cost, and readily available without special tests. Moreover, the preoperative ALBI grade and AAPR score showed greater discriminatory power than Child-Pugh grade. The AAPR was more accurate than ALBI grade. Even among patients with Child-Pugh score 5, they also exhibited their accurate prognostic value in predicting postoperative survival outcome. It was beneficial to consider the ALBI grade and AAPR as useful surrogate markers to identify patients at risk of poor postoperative outcomes, and future prospective validation is required.

\section{DATA AVAILABILITY STATEMENT}

The datasets generated for this study are available on request to the corresponding author.

\section{ETHICS STATEMENT}

The studies involving human participants were reviewed and approved by the ethics committee of the West China Hospital and the Third Affiliated Hospital

\section{REFERENCES}

1. Bray F, Ferlay J, Soerjomataram I, Siegel RL, Torre LA, Jemal A. Global cancer statistics 2018: GLOBOCAN estimates of incidence and mortality worldwide for 36 cancers in 185 countries. CA Cancer J Clin. (2018) 68:39424. doi: $10.3322 /$ caac. 21492

2. Rizvi S. Khan SA, Hallemeier CL, Kelley RK, Gores GJ. Cholangiocarcinoma - evolving concepts and therapeutic strategies. Nat Rev Clin Oncol. (2018) 15:95-111. doi: 10.1038/nrclinonc.2017.157

3. Wang Y. Li J, Xia Y, Gong R, Wang K, Yan Z, et al. Prognostic nomogram for intrahepatic cholangiocarcinoma after partial hepatectomy. J Clin Oncol. (2013) 31:1188-95. doi: 10.1200/JCO.2012.41.5984

4. Saha SK. Zhu AX, Fuchs CS, Brooks GA. Forty-year trends in Cholangiocarcinoma incidence in the US.: intrahepatic disease on the rise. Oncologist. (2016) 21:594-9. doi: 10.1634/theoncologist.2015-0446

5. Shaib Y, El-Serag HB. The epidemiology of cholangiocarcinoma. Semin Liver Dis. (2004) 24:115-25. doi: 10.1055/s-2004-828889

6. Bridgewater J, Galle PR, Khan SA, Llovet JM, Park JW, Patel T, et al. Guidelines for the diagnosis and management of intrahepatic cholangiocarcinoma. $J$ Hepatol. (2014) 60:1268-89. doi: 10.1016/j.jhep.2014.01.021

7. Sonbare DJ. Influence of surgical margins on outcome in patients with intrahepatic cholangiocarcinoma: a multicenter study by the AFC-IHCC-2009 study group. Ann Surg. (2014) 259:e36. doi: 10.1097/SLA.0b013e3182a5c985

8. Edge SB, Compton CC. The American joint committee on cancer: the 7 th edition of the AJCC cancer staging manual and the future of TNM. Ann Surg Oncol. (2010) 17:1471-4. doi: 10.1245/s10434-010-0985-4

9. Lee AJ, Chun YS. Intrahepatic cholangiocarcinoma: the AJCC/UICC 8th edition updates. Chin Clin Oncol. (2018) 7:52. doi: 10.21037/cco.2018.07.03

10. Chun YS. Pawlik TM, Vauthey JN. 8th Edition of the AJCC cancer staging manual: pancreas and hepatobiliary cancers. Ann Surg Oncol. (2018) 25:845-7. doi: 10.1245/s10434-017-6025-x of Sun Yat-sen University. The patients/participants provided their written informed consent to participate in this study.

\section{AUTHOR CONTRIBUTIONS}

HLi, HW, and GW: conceptualization. HLi, JL, and JW: data curation. HLi, BC, and HLiu: formal analysis. GW and HW: supervision. HLi, JL, and JW: writing-original draft. All the authors approved the final version of manuscript.

\section{FUNDING}

This work was supported by National Natural Science Foundation of China (81972747, 81872004, 81800564, 81770615, 81700555 , and 81672882), the Science and Technology Support Program of Sichuan Province (2019YFQ0001, 2018SZ0115, 2017SZ0003), the Science and Technology Program of Tibet Autonomous Region (XZ201801-GB-02) and the 1.3.5 project for disciplines of excellence, West China Hospital, Sichuan University (ZYJC18008).

\section{SUPPLEMENTARY MATERIAL}

The Supplementary Material for this article can be found online at: https://www.frontiersin.org/articles/10.3389/fonc. 2020.00525/full\#supplementary-material

11. Luo X. Yuan L, Wang Y, Ge R, Sun Y, Wei G. Survival outcomes and prognostic factors of surgical therapy for all potentially resectable intrahepatic cholangiocarcinoma: a large single-center cohort study. J Gastrointest Surg. (2014) 18:562-72. doi: 10.1007/s11605-0132447-3

12. Ohtsuka M. Ito H, Kimura F, Shimizu H, Togawa A, Yoshidome H, et al. Results of surgical treatment for intrahepatic cholangiocarcinoma and clinicopathological factors influencing survival. Br J Surg. (2002) 89:1525-31. doi: 10.1046/j.1365-2168.2002.02268.x

13. Zhang C. Wang $\mathrm{H}$, Ning $\mathrm{Z}$, Xu L, Zhuang $\mathrm{L}$, Wang $\mathrm{P}$, et al. Prognostic nutritional index serves as a predictive marker of survival and associates with systemic inflammatory response in metastatic intrahepatic cholangiocarcinoma. Onco Targets Ther. (2016) 9:6417-23. doi: 10.2147/OTT.S112501

14. Chen Q. Dai Z, Yin D, Yang LX, Wang Z, Xiao YS, et al. Negative impact of preoperative platelet-lymphocyte ratio on outcome after hepatic resection for intrahepatic cholangiocarcinoma. Medicine (Baltimore). (2015) 94:e574. doi: 10.1097/MD.0000000000000574

15. Zhang K. Yu J, Yu X, Han Z, Cheng Z, Liu F, et al. Clinical and survival outcomes of percutaneous microwave ablation for intrahepatic cholangiocarcinoma. Int J Hyperthermia. (2018) 34:292-7. doi: 10.1080/02656736.2017.1327678

16. Chan AW, Chan SL. Albumin-to-alkaline phosphatase ratio: a novel prognostic index for hepatocellular carcinoma. Dis Markers. (2015) 2015:564057. doi: 10.1155/2015/564057

17. Johnson PJ, Berhane S, Kagebayashi C, Satomura S, Teng M, Reeves HL, et al. Assessment of liver function in patients with hepatocellular carcinoma: a new evidence-based approach-the ALBI grade. J Clin Oncol. (2015) 33:550-8. doi: 10.1200/JCO.2014.57.9151

18. Li H. Wang L, Chen L, Zhao H, Cai J, Yao J, et al. Prognostic value of albumin-to-alkaline phosphatase ratio in hepatocellular Carcinoma 
patients treated with Liver transplantation. J Cancer. (2020) 11:2171-80. doi: $10.7150 /$ jca.39615

19. Carlson RV. Boyd KM, Webb DJ. The revision of the Declaration of Helsinki: past, present and future. Br J Clin Pharmacol. (2004) 57:695-713. doi: 10.1111/j.1365-2125.2004.02103.x

20. Johnson PJ. Berhane S, Kagebayashi C, Satomura S, Teng MJ, Skowronska A, et al. Assessment of liver dysfunction in hepatocellular carcinoma (HCC): An international collaborative study. J Clin Oncol. (2014) 32:4094. doi: 10.1200/jco.2014.32.15_suppl.4094

21. Chan AWH. Zhong J, Berhane S, Toyoda H, Cucchetti A, Shi K, et al. Development of pre and post-operative models to predict early recurrence of hepatocellular carcinoma after surgical resection. J Hepatol. (2018) 69:128493. doi: 10.1016/j.jhep.2018.08.027

22. Zheng BH. Yang LX, Sun QM, Fan HK, Duan M, Shi JY, et al. A new preoperative prognostic system combining CRP and CA199 for patients with intrahepatic Cholangiocarcinoma. Clin Transl Gastroenterol. (2017) 8:e118. doi: $10.1038 /$ ctg.2017.45

23. Durand F, Valla D. Assessment of the prognosis of cirrhosis: child-Pugh versus MELD. J Hepatol. (2005) 42 (Suppl. 1):S100-7. doi: 10.1016/j.jhep.2004. 11.015

24. Wang YY, Zhong JH, Su ZY, Huang JF, Lu SD, Xiang BD, et al. Albuminbilirubin versus Child-Pugh score as a predictor of outcome after liver resection for hepatocellular carcinoma. Br J Surg. (2016) 103:725-34. doi: 10.1002/bjs.10095

25. Pinato DJ. Sharma R, Allara E, Yen C, Arizumi T, Kubota K, et al. The ALBI grade provides objective hepatic reserve estimation across each BCLC stage of hepatocellular carcinoma. J Hepatol. (2017) 66:338-46. doi: 10.1016/j.jhep.2016.09.008

26. Grivennikov SI. Greten FR, Karin M. Immunity, inflammation, and cancer. Cell. (2010) 140:883-99. doi: 10.1016/j.cell.2010.01.025

27. Tsilimigras DI, Hyer JM, Moris D, Sahara K, Bagante F, Guglielmi A, et al. Prognostic utility of albumin-bilirubin grade for short- and long-term outcomes following hepatic resection for intrahepatic cholangiocarcinoma: a multi-institutional analysis of 706 patients. J Surg Oncol. (2019) 120:206-13. doi: $10.1002 /$ jso. 25486

28. Cai X, Chen Z, Chen J, Ma X, Bai M, Wang T, et al. Albumin-toalkaline phosphatase ratio as an independent prognostic factor for overall survival of advanced hepatocellular Carcinoma patients without receiving standard anti-cancer therapies. J Cancer. (2018) 9:189-97. doi: 10.7150/jca. 21799
29. Mukaiyama K, Kamimura M, Uchiyama S, Ikegami S, Nakamura Y, Kato H. Elevation of serum alkaline phosphatase (ALP) level in postmenopausal women is caused by high bone turnover. Aging Clin Exp Res. (2015) 27:413-8. doi: 10.1007/s40520-014-0296-x

30. Trauner M, Gulamhusein A, Hameed B, Caldwell S, Shiffman ML, Landis C, The nonsteroidal FXR agonist cilofexor (GS-9674) improves markers of cholestasis and liver injury in patients with primary sclerosing cholangitis. Hepatology. (2019) 70:788-801. doi: 10.1002/hep.30509

31. Zhang C. Wang H, Ning Z, Xu L, Zhuang L, Wang P, et al. Serum liver enzymes serve as prognostic factors in patients with intrahepatic cholangiocarcinoma. Onco Targets Ther. (2017) 10:1441-9. doi: 10.2147/OTT.S124161

32. Liu RQ. Shen SJ, Hu XF, Liu J, Chen LJ, Li XY. Prognosis of the intrahepatic cholangiocarcinoma after resection: hepatitis B virus infection and adjuvant chemotherapy are favorable prognosis factors. Cancer Cell Int. (2013) 13:99. doi: 10.1186/1475-2867-13-99

33. Waked I. Berhane S, Toyoda H, Chan SL, Stern N, Palmer D, et al Transarterial chemo-embolisation of hepatocellular carcinoma: impact of liver function and vascular invasion. Br J Cancer. (2017) 116:448-54. doi: $10.1038 /$ bjc. 2016.423

34. Hickey R, Mouli S, Kulik L, Desai K, Thornburg B, Ganger D, et al. Independent analysis of albumin-bilirubin grade in a 765-patient cohort treated with transarterial locoregional therapy for hepatocellular carcinoma. J Vasc Interv Radiol. (2016) 27:795-802. doi: 10.1016/j.jvir.2016. 03.005

35. Oh IS, Dong HS, Kang TW, Min WL, Kang W, Gwak GY, et al. Liver function assessment using albumin-bilirubin grade for patients with very early-stage hepatocellular Carcinoma treated with radiofrequency ablation. Dig Dis Sci. (2017) 62:3235-42. doi: 10.1007/s10620-017-4775-8

Conflict of Interest: The authors declare that the research was conducted in the absence of any commercial or financial relationships that could be construed as a potential conflict of interest.

Copyright (c) $2020 \mathrm{Li}, \mathrm{Li}$, Wang, Liu, Cai, Wang and Wu. This is an open-access article distributed under the terms of the Creative Commons Attribution License (CC $B Y)$. The use, distribution or reproduction in other forums is permitted, provided the original author(s) and the copyright owner(s) are credited and that the original publication in this journal is cited, in accordance with accepted academic practice. No use, distribution or reproduction is permitted which does not comply with these terms. 